\title{
Management of Aortic Dissection in Marfanoid young patient with Ebstein anomaly: Rare Case Report
}

\author{
Article by Naresh $\operatorname{Sen}^{1}$ and Sonal Tanwar ${ }^{2}$ \\ ${ }^{1}$ Narayana Hrudayalaya Institute of Cardiac Sciences, Bangalore (India) \\ Email id: drnaresh.sen@gmail.com \\ ${ }^{2}$ Anand Multispeciality Hospital, Beawar, Rajasthan (India) \\ Email id: drtanwarsonal@gmail.com
}

\begin{abstract}
Background: Marfan syndrome is an autosomal dominant connective tissue disorder characterized by a combination of clinical manifestations in different organ systems. Patients with Marfan syndrome (MFS) whose lifetimes are extended may be encountered as acute abdomen cases (Aortic dissection) apart from the obligatory reasons and emergencies arising naturally out of their disease, as in the case reported. Ebstein's anomaly is a rare congenital heart disease; in which there is a downward displacement of the tricuspid valve into the right ventricle.

Case Report: 25-year-old Indian male shopkeeper, $47 \mathrm{~kg}$ in weight and $176 \mathrm{~cm}$ tall was admitted in emergency department with acute abdominal pain associated with dyspnea. Long arms and legs, arachnodactyly, kyphoscoliosis, pectus excavatum were found to be present in the physical examination. According to chest radiography, density increase in the left apical field (hyperluscency) (thought to be secondary to bullous structure), transpiring in the ascendent aorta, a rightward shift of the heart (due to the effect of the kyphoscoliosis and pectus excavatum), and thoracolumbar scoliosis (kyphoscoliosis) with a rightward aperture were detected. In addition, according to the echocardiographic examination, Ebstein's anomaly, Aortic Dissection, Tricuspid Regurgitaion, pulmonary hypertension, and inferior deficiency of mitral, aorta, and tricuspid valves were present. Moreover, a right bundle block (secondary to Ebstein's anomaly) was observed in the electrocardiographic examination. Finally he was shifted to cardiothoracic surgery unit for surgical repair of aortic dissection and ebstein anomaly.

Conclusion: For cases of Marfan Syndrome (MFS), the risks of which have been detected through a detailed cardiac examination including echocardiography, we believe that they can be operated as emergency cases with close anesthesia management. Mostly ascending aorta is involved with marfan syndrome but in this case evidence of ebstein anomaly is an interesting point towards cardiovascular surgery and learning about management and post op care.
\end{abstract}

Keywords: Aortic dissection, marfan syndrome, ebstein anomaly.

\section{Introduction}

Marfan syndrome is an autosomal dominant connective tissue disorder characterized by a combination of clinical manifestations in different organ systems. Patients with Marfan syndrome (MFS) whose lifetimes are extended may be encountered as acute abdomen cases (Aortic dissection) apart from the obligatory reasons and emergencies arising naturally out of their disease, as in the case reported. The gene that causes MFS, FBN1, is located on chromosome 15 and encodes fibrillin-1. The condition affects the skeletal, skin/integumentary, ocular, pulmonary, and cardiovascular systems. (1) Although MFS is a multisystem disorder, the most clinically significant manifestation is cardiovascular disease. Because of the abnormal supporting tissues of the aorta and cardiac valves, individuals with MFS are predisposed to the development of aortic dilatation and valvular insufficiency. Mitral valve prolapse with regurgitation is common and can lead to the development of endocarditis 
South American Journal of Medicine

Volume 4, Issue 1, 2016

or congestive heart failure. The natural history of the disease includes a substantial risk of sudden and premature death from aortic dissection. Life expectancy is almost halved in patients with MFS, cardiovascular complications being responsible for $95 \%$ of deaths. However, with rapid advances in medical and surgical treatments, together with reduction of physical and hemodynamic stress, effective management of patients has improved life expectancy. Serial imaging of the aorta is suggested, with MRI being the preferred imaging technique. In an acute dissection treatment choice depends on its location. For Stanford type A (ascending aortic) dissection, surgical management is superior to medical management. For uncomplicated Stanford type B (distal aortic) dissections (including abdominal aortic dissections), medical management is preferred over surgical. $(2,5)$

Ebstein's anomaly is a rare congenital heart disease; in which there is a downward displacement of the tricuspid valve into the right ventricle. It has a distal effective portion and an atrialized proximal portion(3). It is frequently associated with intracardiac shunting, pulmonary hypertension, cardiac dysrhythmias and cyanosis. Clinically, there is a wide spectrum of severity of disease with patients ranging from those who are asymptomatic to those who are debilitated ${ }^{1}$. Congestive heart failure and sudden collapse are the most common causes of death. The incidence in general population is $1: 110,000$ and there is no sex difference. $(3,4)$

\section{Case report}

The case was 25 -year-old Indian male shopkeeper, $47 \mathrm{~kg}$ in weight and $176 \mathrm{~cm}$ tall. He has admitted in emergency department with acute abdominal pain associated with dyspnea and chest heaviness. Tall and slender build, Disproportionately fingers (Figure-1), long arms and legs, arachnodactyly, kyphoscoliosis, pectus excavatum were found to be present in the physical examination.

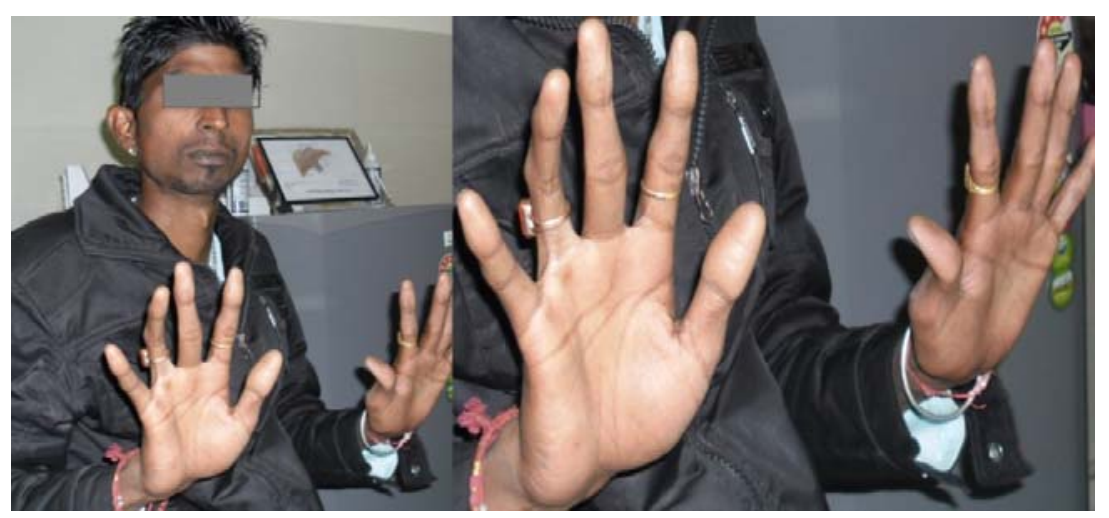

Figure-1 (Disproportionately fingers)

His Vital Sign revealed Blood pressure was $134 / 86 \mathrm{mmHg}$, temperature $-98.2^{\circ} \mathrm{F}\left(36.8^{\circ} \mathrm{C}\right)$; heart rate $-68 \mathrm{bpm}$ and respiratory rate was 26 breaths/min. Raised JVP, regular heart rhythm with no extra sounds, soft S1, grade 4/6 systolic murmur in the lower parasternal area and the absence of thrill. The lungs auscultation revealed bilaterally fine basal crepts. with an oxygen saturation of $94 \%$. The abdomen is nondistended, soft, and tender in epigastric region, with no palpable masses. There is no pedal edema of the extremities. The neurologic examination reveals normal mentation, intact cranial nerves, intact motor strength and sensation, and normal reflexes. No tremor is noted. Moreover, a right bundle block (secondary to Ebstein's anomaly) was observed in the electrocardiographic examination(Figure-2). In addition, according to the echocardiographic examination, Ebstein's anomaly, Aortic Dissection, Tricuspid Regurgitaion, pulmonary hypertension, and inferior deficiency of mitral, aorta, and tricuspid valves were present(Figure-3). According to chest radiography, density increase in the left apical field (hyperluscency) (thought to be secondary to bullous structure), transpiring in the ascending aorta, a rightward shift of the heart (due to the effect of the kyphoscoliosis 
and pectus excavatum), and thoracolumbar scoliosis (kyphoscoliosis) with a rightward aperture were detected (Figure-4).

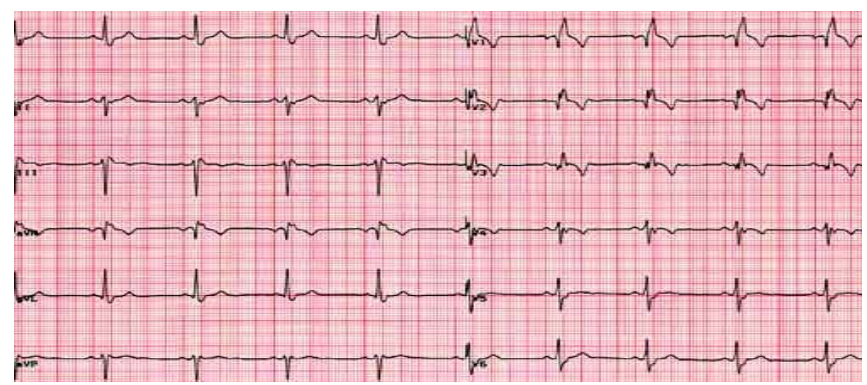

Figure-2 (ECG- Right Bundle Branch Block.)
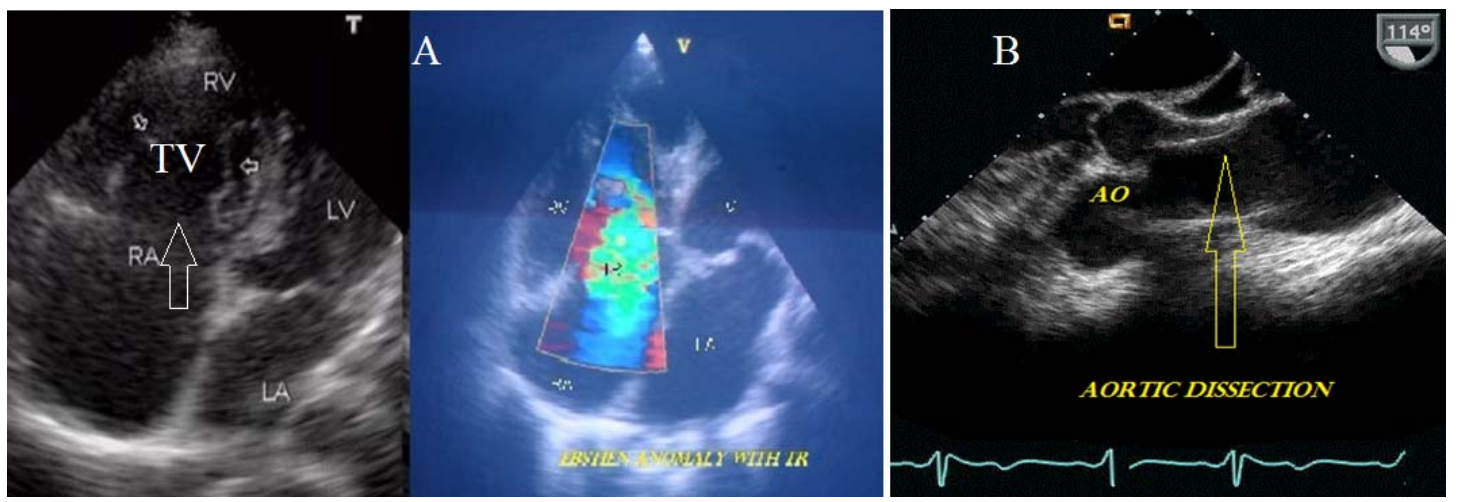

Figure-3 (A. 2D Transthorasic Echo- Ebstein Anomaly with TR, B. 2D Trans Esophageal Echo- Aortic Dissection

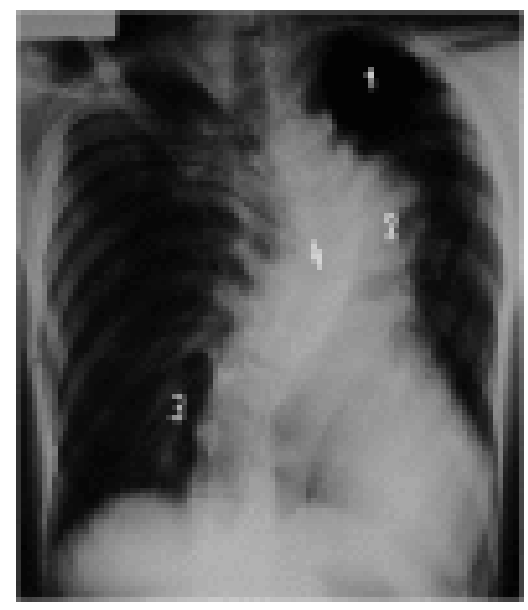

Figure-4 (X-ray during preoperative preparation. 1, density increase in the left apical area; 2, transpiring in ascendent aorta; 3 , a rightward shift of the heart; 4 , scoliosis)

During the preoperative laboratory examination no other pathology was detected besides leucocytosis $\left(17 \times 10^{9} / \mathrm{L}\right)$, CRP elevation $(16.2 \mathrm{mg} / \mathrm{dL})$, and albumin decrease $(31 \mathrm{~g} / \mathrm{L})$. Preoperative blood gas values were in the normal range. A moving and soft tissue structure (connection with decreasing aortic dissection) disturbing phleb of the aorta lumen inside the composition of thoracic descending aorta and abdominal aorta was observed in the abdominal ultrasonography with confirmation support the presence of acute aorta dissection were found in tomographic examination. (Figure-5) 
South American Journal of Medicine

Volume 4, Issue 1, 2016

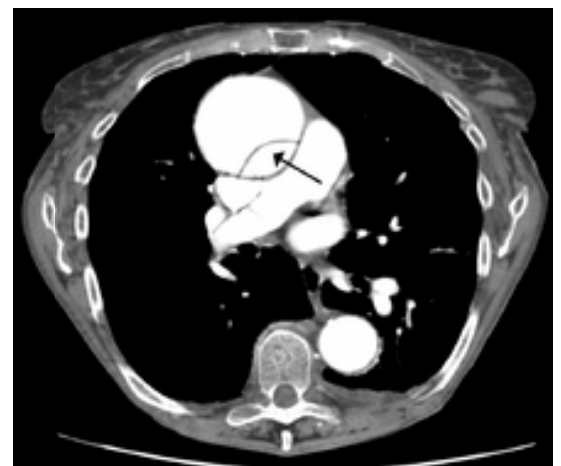

Figure-5 (Aortic dissection, Type A II - Originates in ascending aorta and is confined to the ascending aorta)

The patient was being monitored by the cardiology outpatient clinic due to MFS and Ebstein's anomaly. The prescribed drugs were metoprolol, digoxin, and alprazolam. More importantly, previous detailed genetic research on the patient showed that there was a mutation in the FBN1 gene on the 15th chromosome. Finally he was shifted to cardiothorasic sugery unit for surgical repair of aortic dissection and ebstein anomaly.

\section{Short comments on treatment part.}

\subsubsection{Repairing type a dissections of aorta.}

Patients with type A dissections are treated with immediate surgical correction. This involves transfer to the operating room, where median sternotomy is performed. Profound hypothermia is initiated after the patient is placed on cardiopulmonary bypass. Cardiopulmonary bypass is performed by femoral-femoral cannulation and through the superior vena cava for retrograde cerebral perfusion. In an acute dissection treatment choice depends on its location. For Stanford type A (ascending aortic) dissection, surgical management is superior to medical management. For uncomplicated Stanford type B (distal aortic) dissections (including abdominal aortic dissections), medical management is preferred over surgical. (7)

\subsubsection{Medication for aortic dissections}

Aortic dissection generally presents as a hypertensive emergency, and the prime consideration of medical management is strict blood pressure control. The target blood pressure should be a mean arterial pressure (MAP) of 60 to $75 \mathrm{mmHg}$, or the lowest blood pressure tolerated by the patient. Another factor is to reduce the shear-force $\mathrm{dP} / \mathrm{dt}$ (force of ejection of blood from the left ventricle). Beta blockers are first line treatment for patients with acute and chronic aortic dissection. In acute dissection, rapidly acting, titratable parenteral agents (such as esmolol, propranolol, or labetalol) are preferred. Vasodilators such as sodium nitroprusside can be considered for patients with ongoing hypertension, but they should never be used alone, as they generally cause reflex tachycardia. Calcium can be used in the treatment of aortic dissection, particularly if there is a contraindication to the use of beta blockers. The calcium channel blockers typically used are verapamil and diltiazem, because of their combined vasodilator and negative inotropic effects. $(\mathbf{7 , 8})$

\subsection{Surgery for ebstein anomaly}

Surgical care includes correction of the underlying tricuspid valve and right ventricular abnormalities, correction of any associated intracardiac defects, palliative procedures in early days of life as a bridge to more definitive surgical treatment later, and surgical treatment of associated arrhythmias. Complete repair of Ebstein anomaly in symptomatic neonates has been shown to be feasible, with good early and late survival and excellent functional status. In patients $\geq 50$ years of age with Ebstein anomaly, surgery is associated with good long-term 
survival and improved functional status, although long-term survival might be improved by performing surgery earlier. (13)

The trend is to perform surgery earlier rather later in the course of heart failure. Indications for surgery are generally as follows: New York Heart Association (NYHA) class I-II heart failure with worsening symptoms or with a cardiothoracic ratio of 0.65 or greater. (14) NYHA class III-IV heart failure, History of paradoxical embolism, Significant cyanosis with arterial $\mathrm{O}_{2}$ saturation of $80 \%$ or less and/or polycythemia with hemoglobin of $16 \mathrm{~g} / \mathrm{dL}$ or more and Arrhythmias refractory to medical and radiofrequency ablation

Various approaches are available to treat structural abnormalities. Tricuspid valve repair is preferred over valve replacement, and bioprosthetic valves are preferred over mechanical prosthetic valves. The atrialized portion of the right ventricle can be resected surgically, and the markedly dilated, thin-walled right atrium can be resected. Associated septal defects may be closed. Palliative procedures include creation of atrial septal defect, closure of tricuspid valve with plication of the right atrium, and maintenance of pulmonary blood flow through aortopulmonary shunt. Palliative procedures usually are reserved for severely ill infants with otherwise dire prognosis.

Left ventricular dysfunction should not be considered a contraindication to tricuspid valve surgery. In these patients, although early mortality is greater with tricuspid valve surgery, the late results are favorable and left ventricular function seems to improve postoperatively. $(\mathbf{1 5 , 1 6 )}$ Functional status improves after surgery. Surgical treatments of arrhythmias include the following: Ablation of the accessory pathways and Maze procedure for atrial arrhythmias. Cardiac transplantation is appropriate in selected patients.

\section{Discussion}

MFS primarily affects cardiovascular, skeletal, ocular, integumentary and pulmonary systems. Cardiovascular complications are of special importance as they constitute the primary cause of mortality. The most serious medical complication of MFS is dilation and dissection of the ascending aorta, which can lead to aortic rupture and premature death if untreated. But management routinely includes use of beta-adrenergic blockers, which have been shown to slow the progressive widening of the aorta in many patients with MFS. Other cardiovascular complications include mitral valve prolapse, aortic regurgitation, tricuspid valve prolapse, and dilation of the main pulmonary artery. (1)

The skeletal manifestations of the condition include tall stature; thin habitus with increased arm span-to-height ratio; long, slender fingers (arachnodactyly); elongated face; high arched palate; pes planus; joint laxity; scoliosis; and pectus deformity. Ectopia lentis (lens dislocation) is a hallmark ocular feature present in approximately $60 \%$ of patients. Other ocular findings include corneal flatness, increased axial globe length leading to nearsightedness, and hypoplastic irides. Spontaneous pneumothorax or apical blebs are the most common pulmonary abnormalities. Other cardinal features involve skin, integument, and dura, and include striae atrophicae (i.e., stretch marks), recurrent hernias, and lumbosacral ectasia. (2)

The progressive and potentially fatal clinical features of MFS make early diagnosis very important. Due to the lack of a molecular diagnostic test, diagnosis depends on the revised (Ghent) clinical criteria. The most prominent major criteria are: a constellation of skeletal manifestations including pectus carinatum or excavatum, reduced upper- to-lower-segment ratio, or arm-span-to-height ratio $>1.05$, scoliosis, and reduced elbow extension; ectopia lentis; dilatation or dissection of the ascending aorta; lumbosacral dural ectasia; and inheritance of a genotype previously associated with classic MFS or an unequivocal family history. Spontaneous pneumothorax, striae atrophicae, and recurrent hernias are also useful signs for diagnosis. Diagnostic dilemmas arise due to considerable inter- and intrafamilial variability. (5)

Cardiovascular assessment is mandatory before embarking on surgery due to the threat to life from mitral valve prolapse and aortic dilatation. Blood pressure may be maintained below 
South American Journal of Medicine

Volume 4, Issue 1, 2016

normal with antihypertensive agents. Individuals are at risk from spontaneous pneumothorax, usually arising out of an apical bulla, which may complicate a present compromise. The major objectives of surgery for aortic dissection are to alleviate the symptoms, decrease the frequency of complications, and prevent aortic rupture and death. The affected layers of the aorta are sutured together, and the aorta is reinforced with a Dacron graft. Improved cardiopulmonary bypass circuits have decreased the prevalence of injury to blood elements. Morbidity and mortality rates associated with this highly invasive surgery have decreased with the introduction of profound hypothermic circulatory arrest and retrograde cerebral perfusion. (7)

Dissections involving the arch are more complicated than those involving only the ascending aorta because the innominate, carotid, and subclavian vessels branch from the arch. Deep hypothermic arrest is usually required. If the arrest time is less than 45 minutes, the rate of CNS complications is less than $10 \%$. Retrograde cerebral perfusion may improve the protection of the CNS during the arrest period. The mortality rate associated with aortic arch dissections is approximately $10-15 \%$. Significant neurologic complications occur in an additional $10 \%$ of patients.

Postoperative complications for extensive disease involving the thoracoabdominal aorta include myocardial infarction, respiratory failure, renal failure, stroke, and paraparesis or paraplegia. The use of adjunct procedures has decreased the frequency of procedure-related spinal cord injury during descending aorta and thoracoabdominal surgeries. The long term follow-up in individuals who survive aortic dissection involves strict blood pressure control. The relative risk of late rupture of an aortic aneurysm is 10 times higher in individuals who have uncontrolled hypertension, compared to individuals with a systolic pressure below 130 mmHg. (9) Management of anesthesia for MFS has specific precautions for valvular diseases, thoracic aortic aneurysm, and skeletal joint laxity. Patients with MFS must be carefully assessed preoperatively for specific complications. Antibiotic prophylaxis should be given before dental and other surgical procedures. The anesthesiologist should be prepared for a potentially difficult intubation. (9)

No single intraoperative anesthetic agent or technique has demonstrated superiority. But the anesthetic technique chosen should not decrease myocardial contractility and should avoid sudden increases in contractility, in order to minimize the risk of aortic dissection or rupture. Perioperative hypertension or hypotension must be avoided. The presence of significant aortic insufficiency warrants that the blood pressure be high enough to provide adequate coronary blood flow but should not be so high as to risk dissection of the aorta. Preoperative echocardiography should be considered so as to exclude cardiac or aortic pathology. A patient has been reported who had acute, intraoperative coronary artery obstruction (presumably not a coronary air embolus). (10)

Patients may have significant pulmonary problems including restriction of lung function due to pectus excavatum, kyphoscoliosis, or intrinsic pulmonary involvement with emphysema. Bronchogenic cysts and "honeycomb lung" that lead to spontaneous pneumothorax must also be kept in mind when using positive pressure ventilation. Midtracheal obstruction has been reported after Harrington rod placement and secondary to unexpected tracheomalacia after the induction of general anesthesia. Patients must be carefully positioned to avoid joint dislocations secondary to joint laxity. Although there is a possibility of temporomandibular joint dysfunction, this has not been reported to cause difficulty with laryngoscopy. At extubation, care should be taken to avoid sudden increases in blood pressure or heart rate. Adequate postoperative pain management is vitally important to avoid detrimental hypertension and tachycardia. (8-11)

Ebstein's anomaly includes an abnormal tricuspid valve and "atrialized" right ventricle, that is, the septal leaflets and often the posterior leaflets of the tricuspid valve are displaced into the right ventricle. But the anterior leaflet is usually malformed, excessively large, and abnormally attached or adherent to the right ventricular free wall. Thus, a portion of the right ventricle is "atrialized" in that it is located on the atrial side of the tricuspid valve, and the 
remaining functional right ventricle is small. Eighty percent of patients with Ebstein's anomaly have an interatrial communication (atrial septal defect or patent foramen ovale) through which right-to-left shunting of blood may occur. (3) The clinical presentatation of Ebstein's anomaly varies greatly. As in our case if the tricuspid valve is minimally displaced, tricuspid regurgitation may actually be mild enough to go unrecognized until adulthood. In fact, the diagnosis may be made by accident with few, if any symptoms. The electrocardiogram will show evidence of right bundle block or right atrial enlargement in over $50 \%$ of the cases. $(3,4)$

Anesthesia on patients with Ebstein's anomaly depends to a large degree on the clinical manifestations. The right ventricular dysfunction which puts these patients especially at a high risk for anesthesia is worsened by the tricuspid regurgitation. Similarly, poor left ventricular function is an especially ominous feature before anesthesia. One must be very observant in Ebstein's anomaly patients during the perioperative period for the occurrence of arrhythmias (especially supraventricular arrhythmias such as Wolff- Parkinson-White syndrome). It is important to review the chronic antiarrhythmic medications that the patient may be receiving. (4)

Prior monitoring and treatment of the case by the cardiology clinic positively affected cardiac performance. Moreover, careful preoperative evaluation, a good preparation, close anesthesia management, and postoperative care prevented fatal complications from developing in the patient. (13-15)

\section{Problem faced and lesion learnt}

At the end of the operation, the patient, in whom cutaneous and subcutaneous local anesthetic infiltrations were applied, was extubated to reverse the neuromusculer blocking effect while nitroglycerine infusion continued. While the patient was recovering consciousness, he was taken to the intensive care unit (ICU) for monitoring and intravenously patient-controlled analgesia and nitroglycerine infusion continued. The patient was observed in the ICU for 2 days after the operation and was transferred to the surgical clinic without any complications. The case was discharged from the hospital on the sixth postoperative day. $(8,9)$

\section{Conclusion}

For cases of Marfan Syndrome (MFS), the risks of which have been detected through a detailed cardiac examination including echocardiography, we believe that they can be operated as emergency cases with close anesthesia management. Mostly ascending aorta is involved with marfan syndrome but in this case evidence of ebstein anomaly is an interesting point towards cardiovascular surgery and learning about management and post op care. The major objectives of surgery for aortic dissection are to alleviate the symptoms, decrease the frequency of complications, and prevent aortic rupture and death. The affected layers of the aorta are sutured together, and the aorta is reinforced with a Dacron graft. In case of ebstein anomaly, surgical care includes correction of the underlying tricuspid valve and right ventricular abnormalities, correction of any associated intracardiac defects, palliative procedures in early days of life as a bridge to more definitive surgical treatment later, and surgical treatment of associated arrhythmias.

\section{Consent}

We have taken consent from the patient for the pictures provided.

\section{Acknowledgements}

I would like to thank to Prof. George cherian HOD in Cardiology at Narayana Hrudayalaya, Bangalore who gave support and concern about learning and also I thank to Texila American University or Academic department for continuous learning. 
South American Journal of Medicine

Volume 4, Issue 1, 2016

\section{References}

[1.] Ades L. Guidelines for the diagnosis and management of Marfan syndrome. Heart Lung Circ. 2007;16:28-30.

[2.] Attenhofer Jost CH, Connolly HM, Scott CG, Burkhart HM, Warnes CA, Dearani JA. Outcome of cardiac surgery in patients 50 years of age or older with Ebstein anomaly: survival and functional improvement. J Am Coll Cardiol. 2012 Jun 5. 59(23):2101-6.

[3.] Attie F, Casanova JM, Zabal C, Buendía A, Miranda I, Rijlaarsdam M. Ebstein's anomaly. Clinical profile in 174 patients. Arch Inst Cardiol Mex. 1999 Jan-Feb. 69(1):17-25..

[4.] Brickner ME, Hillis LD, Lange RA. Congenital heart disease in adults: second of two parts. N Engl J Med. 2000;342:334-342.

[5.] Brown ML, Dearani JA, Danielson GK, Cetta F, Connolly HM, Warnes CA, et al. Effect of operation for Ebstein anomaly on left ventricular function. Am J Cardiol. 2008 Dec 15. 102(12):17247.

[6.] Brown ML, Dearani JA, Danielson GK, Cetta F, Connolly HM, Warnes CA, et al. Functional status after operation for Ebstein anomaly: the Mayo Clinic experience. J Am Coll Cardiol. 2008 Aug 5. 52(6):460-6.

[7.] Dean JCS. Management of Marfan syndrome. Heart. 2002;88:97-103.

[8.] Groves ER. Epidural analgesia for labour in a patient with Ebstein's anomaly. Can J Anaesth 1995;42(1):77-79.

[9.] McGoldrick KE. Eye, ear, nose, and throat diseases. In: Fleisher LA, editor. Anesthesia and Uncommon Disease. 5th ed. Philadelphia: Saunders Elsevier; 2006. pp. 6-7.

[10.] Meijboom LJ, Nolle GJ, Mulder BJM. Prevention of cardiovascular complications in the Marfan syndrome. Vas Disc Prevent. 2004;1:79-86.

[11.] Mesrobian RB, Epps JL. Midtracheal obstruction after Harrington rod placement in a patient with Marfan's syndrome. Anesth Analg. 1986;65:411-413.

[12.] Misa VS, Pan PH. Evidence based case report for analgesic and anaesthetic management of parturient with Ebstein's anomly and Wolff-Parkinson- White syndrome. Int J Obstet Anesth 2007;16:77-81.

[13.] Oh AY, Kim YH, Kim BK, et al. Unexpected tracheomalacia in Marfan syndrome during general anesthesia for correction of scoliosis. Anesth Analg. 2002;95:331-332.

[14.] Oliver WC, Lynch JJ. Congenital heart disease. In: Fleisher LA, editor. Anesthesia and Uncommon Disease. 5th ed. Philadelphia: Saunders Elsevier; 2006. pp. 115-118.

[15.] Robinson PN, Godfrey M. The molecular genetics of Marfan syndrome and related microfibrillopathies. J Med Genet. 2000;37:9-25.

[16.] Shores J, Berger KR, Murphy EA, Pyeritz RE. Progression of aortic dilatation and the benefit of long-term beta-adrenergic blockade in Marfan's syndrome. N Engl J Med. 1994;330:1335-1341. 